\title{
Bioequivalence Studies of Generic Metformin on Healthy Volunteers in Nigeria
}

\author{
Awobusuyi Olugbenga* \\ Obafemi Awolowo University, Nigeria \\ *Corresponding Author: Awobusuyi Olugbenga, Obafemi Awolowo University, Nigeria.
}

Received: April 26, 2019; Published: June 07, 2019

DOI: $10.31080 /$ ASPS.2019.03.0307

Diabetes is a disease with a huge economic burden and morbidity globally. However, with developments in medicine and treatment of diabetes, oral antidiabetics have fast become a standard treatment for type 2 diabetes. Metformin is the only remaining biguanide oral antidiabetic drug in the market. Among all the oral antidiabetics in use, metformin stands out for some interesting reasons. Metformin is cardioprotective and has been shown to be superior to other oral antidiabetics in lowering macrovascular and microvascular complications that can arise as a result of prolonged uncontrolled blood sugar in diabetes. However, this evidence has been challenged recently by some researchers claiming that the methodology of the studies was poor. It is also thought that metformin has the tendency to cause weight loss. Hence, it is usually preferred in obese people with type 2 diabetes as opposed to sulfonylureas which can cause weight gain as a side effect. However, metformin can be given both to obese and non-obese people with type 2 diabetes. Perhaps, it is all these factors that makes metformin one of the preferred oral antidiabetic medications in the treatment of type 2 diabetes. It comes in $500 \mathrm{mg}, 850 \mathrm{mg}$ and $1000 \mathrm{mg}$ tablets which can be given once or twice daily depending on the formulation. While innovator brands of metformin are the gold standard in the market, they tend to be too expensive. This significantly raises the financial burden which is felt more among the middle class and lower income class. In an effort to reduce this burden, generic metformin brands can be used in place of innovator brands. However, in order to prevent therapy failure, there is a need to compare the pharmacokinetic profile of generic brands against the innovator brands. This ensures that the cheaper generic brands have similar pharmacokinetic profiles to the more expensive innovator brands. This ensures quality pharmaco-economics and improves pharmaceutical care.

In order to achieve this, we passed different brands of metformin through various tests which conform to the British and United States pharmacopoeia. These tests include dissolution tests, weight uniformity tests, disintegration tests and finally bioequivalence test. It is important that all brands pass all these tests and are similar to the innovator brand both in the dissolution tests and in the bioequivalence tests. In most cases however, there is a biowaiver for the bioequivalence test if the generic brand is found to be similar to the innovator brand during the dissolution tests. This is because the dissolution phase is the phase wherein the active pharmaceutical ingredient is released from the formulation. Out of 14 different brands tested, only 10 conformed to the weight uniformity test. Furthermore, only eight generic brands passed the dissolution test by releasing $75 \%$ within 45 minutes. However, only three generic products met the expected specification of releasing $85 \%$ of their API within 15 minutes. Unfortunately, the innovator brand was not among. Among the three successful generic brands, only one was selected for a bioequivalence comparison with the innovator brand of metformin due to financial constraints. It was found to be pharmacokinetically bioequivalent to the innovator brand of metformin on assessment.

The clinical implications of running bioequivalent tests lies in the fact that the pharmacokinetic profile of a drug indicates its onset, duration, dosing and possibility of pharmacotherapeutic success or failure. Cheaper generics with similar pharmacokinetic profiles to more expensive innovator brands can serve as an alternative therapy to the low-income class. This reduces the financial burden of diabetes and can improve pharmaco-economics of oral antidiabetic medications and adherence to medications due to increased affordability.

\section{Volume 3 Issue 7 July 2019 \\ (C) All rights are reserved by Awobusuyi Olugbenga.}

